\title{
Dinamometria lombar: um teste funcional para o tronco
}

\author{
Lumbar dynamometry: a functional test for the torso
}

\author{
Fernando Luís Fischer Eichinger', Antonio Vinicius Soares², José Marques de Carvalho Júnior², \\ Monique da Silva Gevaerd ${ }^{3}$, Susana Cristina Domenech ${ }^{3}$, Noé Gomes Borges Júnior ${ }^{3}$
}

\begin{abstract}
RESUMO | Contexto: A força muscular é a principal valência física. Sua avaliação clínica é realizada utilizando-se testes manuais. Esses testes, embora reprodutíveis, são muito subjetivos e pouco precisos. A avaliação do tronco vem sendo realizada por meio da dinamometria lombar (DL). Essa informação é valiosa nos exames admissionais e periódicos de colaboradores que atuam em tarefas que exigem esforço repetitivo e manejo de cargas de levantamento e transferência. Objetivos: Como a literatura não possui valores referenciais para esse teste, buscamos apresentar valores que podem ser utilizados como referência para futuros estudos de valores normativos. Métodos: O estudo foi conduzido com 691 participantes saudáveis de ambos os sexos, praticantes e não praticantes de atividade física regular, todos com índice de massa corporal (IMC) normal. A faixa etária variou entre 18 e 45 anos, sendo 382 homens, com idade média de 27,1 anos $( \pm 7,5)$, e 309 mulheres, com idade média 28,2 anos $( \pm 8,1)$. Foi utilizado um sistema de dinamometria digital portátil composto por uma plataforma, conectada a uma célula de carga por meio de uma corrente, e um indicador digital. Resultados: O valor médio de força medido com o sistema de DL, em homens, foi $114,0 \mathrm{kgf}( \pm 25,4)$, e 48,5 kgf $( \pm 18,8)$ nas mulheres. Portanto, a amplitude de força exercida pelos homens foi $235,1 \%$ superior à exercida pelas mulheres. Conclusão: Esse estudo apresenta uma contribuição inicial para o estabelecimento de valores normativos desse teste dinamométrico; porém, outros estudos são necessários, uma vez que as medições realizadas são preliminares e específicas para a região geográfica estudada.
\end{abstract}

Palavras-chave I força muscular; avaliação; região lombar; tronco.

ABSTRACT | Context: Muscle strength is the main physical valence. Clinical evaluation is performed using manual tests. However, these tests - although reproducible - are very subjective and imprecise. The torso evaluation has been performed by means of the the lumbar dynamometry (LD). This information is valuable in admission and periodic examinations of employees working on tasks that require repetitive effort, lifting, and transfer of loads. Objectives: As literature does not have reference values for this test, the objective of this research was to present values that can be used as reference for future study of normative values. Methods: The study was conducted with 691 healthy participants of both the genders and practitioners that do not practice regular physical activity, all with normal body mass index (BMI). Their ages ranged between 18 and 45 years, 382 were men, with an average age of 27.1 years $( \pm 7.5)$, and 309 women, with an average age of 28.2 years $( \pm 8.1)$. A portable digital system is composed of a dynamometer connected to a load cell and platform, and a digital indicator. Results: The average value of force measured in men with the LD system was $114.0 \mathrm{kgf}$ ( \pm 25.4$)$, and $48.5 \mathrm{kgf}( \pm 18.8)$ in women. Men had 235,1\% more strength than women. Conclusion: This study presents an initial contribution to establish normative values for this dynamometer test, but further studies are needed, given the fact that the measurements are preliminary and specific to the geographic region studied.

Keywords I muscle strength; evaluation; lumbosacral region; torso.

Trabalho realizado na Associação Catarinense de Ensino (ACE), Faculdade Guilherme Guimbala (FGG) - Joinville (SC), Brasil.

IUniversidade do Estado de Santa Catarina (CEFID/UDESC) - Florianópolis (SC), Brasil.

${ }^{2}$ ACE, FGG - Joinville (SC), Brasil.

3Programa de Pós-graduação em Ciências do Movimento Humano, CEFID/UDESC - Florianópolis (SC), Brasil.

DOI: 10.5327/Z1679-443520162415 
INTRODUÇÃO

\section{CONTEXTO}

A força muscular (FM) é a capacidade de um músculo ou de um grupamento muscular de gerar torque sobre uma articulação específica ${ }^{1}$. É considerada a valência física mais importante, estando intimamente envolvida com a capacidade funcional ${ }^{2}$.

Uma redução da FM é um preceptor de limitações físicas ${ }^{3,4}$. O declínio na função motora, incluindo um decréscimo na força e no desempenho muscular, leva à redução da autonomia evidenciada na realização das atividades de vida diária (AVDs), principalmente em idosos ${ }^{3}$.

Estudos demonstram que os níveis de FM estão relacionados aos índices de limitações funcionais e problemas de saúde gerais durante a vida ${ }^{5}$, além de estarem independentemente associados com o risco de morte por todas as causas em ambos os sexos e por câncer exclusivamente no sexo masculino ${ }^{6}$.

A fraqueza muscular associada ao uso inadequado do corpo no dia a dia expõe as estruturas da coluna a agravos ${ }^{7}$, o que pode resultar em uma maior taxa de afastamentos temporários ou permanentes do trabalho.

A dor lombar (lombalgia) é uma sintomatologia que afeta em torno de 70 a $85 \%$ dos adultos em algum momento da vida $a^{7,8}$. A lombalgia surge, principalmente, em função do caráter mecânico, como a sobrecarga de força no desenvolvimento das atividades laborais, a permanência por tempo prolongado em posição estática e a repetição de movimentos ${ }^{8}$. Dessa maneira, o fortalecimento da musculatura do tronco pode ser um fator capaz de proporcionar maior proteção nas atividades diárias, sejam elas laborais ou recreacionais.

Mensurar os níveis de força é algo fundamental no que diz respeito à avaliação funcional dos indivíduos. Esse dado é utilizado na prática clínica com diversos objetivos, dentre eles o diagnóstico funcional para avaliação da melhora ou da piora ao longo do tempo, como medida preditiva ou prognóstica para a ocorrência de quedas, limitações funcionais, incapacidades, desabilidades, assim como índices de mortalidade $^{9,10}$.

Como já exposto, a FM possui finalidades em diversas áreas do conhecimento humano, sejam elas científicas ou profissionais.
Diferentes tipos metodológicos de testes podem ser empregados para avaliar os níveis de FM que se caracterizam de acordo com os objetivos do avaliador e a forma de manifestação da força que se pretende monitorar ${ }^{11}$.

A FM isométrica é caracterizada pela atividade muscular, na qual não ocorrem modificações perceptíveis no comprimento das fibras musculares ou no movimento articular visível. Embora não seja realizado trabalho físico, uma grande quantidade de tensão e rendimento de força é produzida pelo músculo ${ }^{11}$.

A avaliação da força por meio de dinamômetros é um procedimento simples, objetivo, prático, de fácil utilização e baixo custo ${ }^{2,3,12}$. Os dinamômetros são equipamentos que permitem a mensuração da força aplicada em um sistema baseado em células de carga ${ }^{13}$. Servem, assim, para estabelecer parâmetros confiáveis na mensuração da força muscular a medida que se torna difícil identificar pequenas variações na graduação desta durante o exame físico ${ }^{14}$.

O dinamômetro é um instrumento muito utilizado para a avaliação da força muscular lombar em indivíduos com estado normal de saúde e/ou em processo de reabilitação ${ }^{15}$. Serve para avaliar a força muscular isométrica (estática) do indivíduo, podendo ser realizados testes de dinamometria torácica, dorsal, dos membros inferiores e de preensão manual ${ }^{16}$.

A utilização da dinamometria lombar (DL) na avaliação da força muscular do tronco é importante para estimar a capacidade física global, sobretudo em sujeitos que realizam grande esforço físico, como atletas ou trabalhadores em atividades ocupacionais que exijam maiores níveis de $\mathrm{FM}^{17,18}$.

Pela importância da avaliação da FM, os clínicos têm cada vez mais interesse na sua investigação. No entanto, habitualmente esses testes de DL são feitos sem considerar valores referenciais, de forma totalmente intuitiva. Assim, profissionais da área da saúde, especialmente médicos e fisioterapeutas, têm realizado com frequência esses procedimentos em serviços de saúde ocupacional, consultórios e clínicas.

\section{OBJETIVOS}

Visto que não existem valores objetivos e normativos para o teste de dinamometria lombar na literatura, este trabalho foi realizado com o objetivo de propor valores normativos para esse teste de força. Visamos dessa forma estabelecer valores 
referenciais para dinamometria lombar, aumentando o conhecimento nessa área específica e incentivando a realização de novos estudos que venham a complementar o presente.

\section{MÉTODOS}

O projeto de pesquisa foi submetido ao Comitê de Ética em Pesquisa da Associação Educacional Luterana Bom Jesus (IELUSC) e aprovado sob o número 015.

O trabalho consiste em um estudo de caráter descritivo com a participação de 691 indivíduos saudáveis de ambos os sexos, praticantes e não praticantes de atividade física regular, todos com o índice de massa corpórea (IMC) normal. A faixa etária variou entre 18 e 45 anos. Foram avaliados 382 homens, com idade média de 27,1 anos $( \pm 7,5)$, e 309 mulheres, com idade média de 28,2 anos $( \pm 8,1)$. Não houve diferença entre os grupos $(p=0,214)$. O presente estudo foi realizado no estado de Santa Catarina, sendo que todos os participantes eram de Joinville e região.
Um questionário foi previamente aplicado com o intuito de coletar todas as informações relevantes para a realização do projeto.

Para a avaliação da força de preensão lombar, foi utilizada uma célula de carga acoplada a uma plataforma de base com corrente e puxador para as mãos. Foi utilizada uma célula de carga CZ FLEXAR ${ }^{\circledast}$, tração/compressão com capacidade de 300,0 kgf e indicador FLEXAR LR-22L ${ }^{\oplus}$, calibrado, com capacidade máxima de 300,0 kgf e mínima de 2,0 kgf, com divisão de $0,05 \mathrm{kgf}$; sensibilidade $0,5 \mu \mathrm{V}$ por divisão.

A Figura 1 ilustra o sistema completo em uso. Para iniciar os testes, o sistema foi ajustado individualmente da seguinte forma: o participante foi posicionado em pé com as mãos apoiadas na região anterior das coxas. $\mathrm{O}$ puxador foi regulado cinco centímetros abaixo dos dedos médios - assim, durante o teste, exigia-se uma semiflexão dos joelhos, evitando uma sobrecarga na região lombar. Cada participante realizou uma tentativa inicial, com intuito de familiarização com o teste. Cada dado registrado representa a média de três medidas.

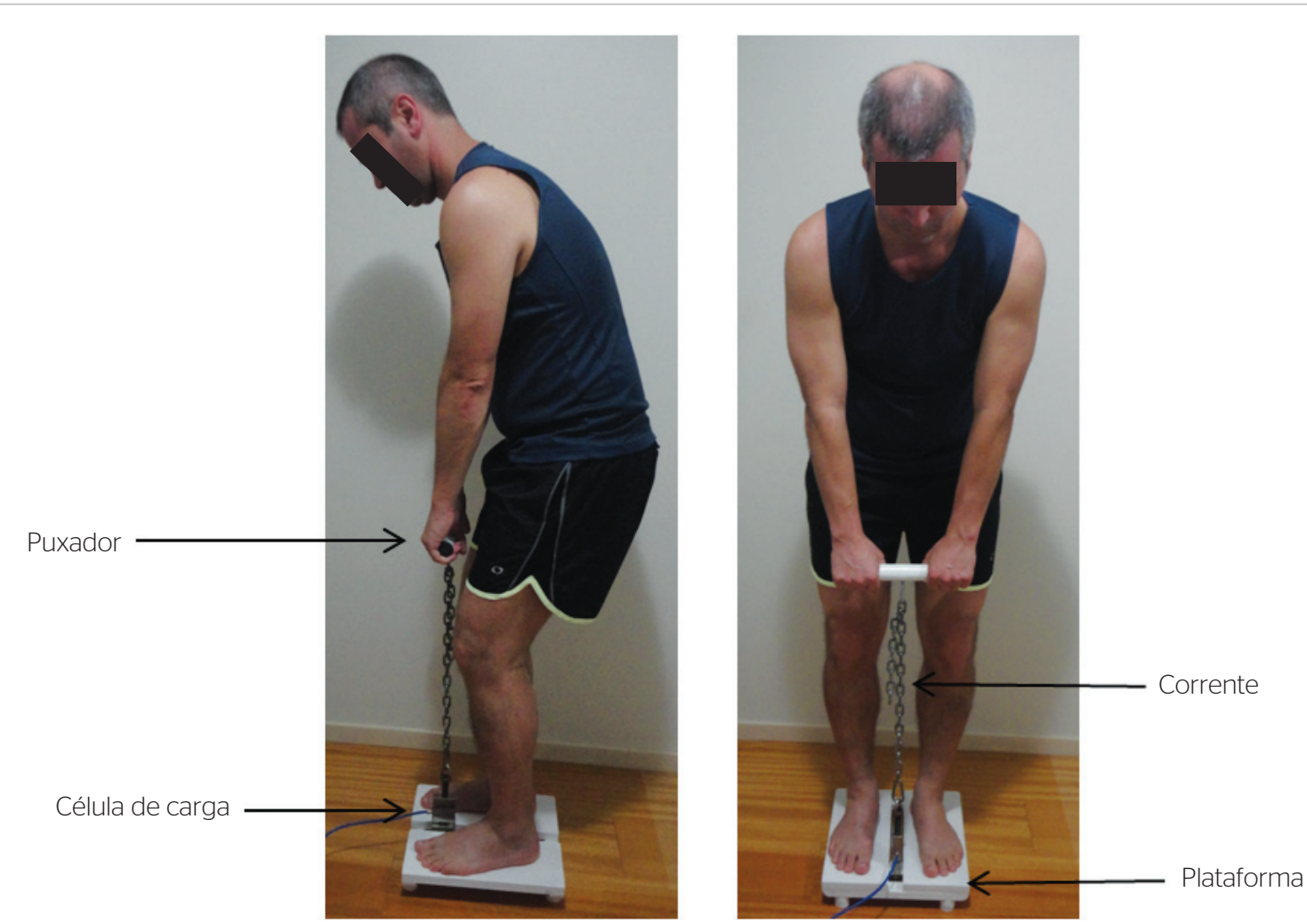

Nota: A fotografia apresenta um dos autores do trabalho demonstrando o uso do sistema completo de dinamometria lombar. A divulgação desta imagem foi autorizada.

Figura 1. Teste de dinamometria lombar. 
Após esclarecimentos e orientações, todos os participantes assinaram o termo de consentimento livre e esclarecido.

As análises incluíram dados de estatística descritiva, como média e desvio padrão.

\section{RESULTADOS}

A Tabela 1 resume a estatística descritiva, apresentando os valores mínimos e máximos, as médias e os desvios padrões da variável analisada, em ambos os sexos.

Neste estudo, constatamos que nos homens a média da força muscular mensurada no teste de DL foi $114,00 \mathrm{kgf}$ $( \pm 25,40)$, enquanto nas mulheres a média foi $48,50 \mathrm{kgf}$ $( \pm 18,80)$. Portanto, a força muscular média mensurada dos participantes do sexo masculino é $235,1 \%$ superior em relação à do gênero feminino. Os detalhes das mensurações estão apresentados na tabela abaixo.

\section{DISCUSSÃO}

A força muscular (FM) é considerada a valência física mais importante do ser humano ${ }^{2}$, sendo uma variável da aptidão física de grande importância ${ }^{19,20,21}$ e relacionada tanto à saúde como ao desempenho físico em diferentes faixas etárias.

A FM pode ser influenciada por elementos internos e externos. Entre os elementos internos destacam-se: a secção transversal da fibra muscular, o número de fibras musculares, a coordenação, a velocidade de contração das fibras musculares, o gênero, o tipo de fibra muscular e a idade. Já entre os elementos externos, pode-se citar a hora do dia, o método de treinamento, a motivação, a nutrição, o doping, entre outros fatores ${ }^{22}$. Outros elementos também interferem no potencial de gerar FM: são os chamados fatores neurais, descritos como o número de unidades

Tabela 1. Resultados dos testes de dinamometria lombar em homens e mulheres.

\begin{tabular}{l|c|c|c|c} 
& Média (kgf) & DP & Mínimo & Máximo \\
\hline Homens (n=382) & 114,00 & 24,50 & 42,50 & 194,00 \\
\hline Mulheres (n=309) & 48,50 & 18,80 & 16,90 & 104,00 \\
\hline
\end{tabular}

kgf: quilograma-força; DP: desvio padrão. motoras ativas, a frequência de disparos e a coativação dos músculos antagonistas ${ }^{23}$.

Estudos mostram que o declínio na função motora, incluindo um decréscimo na força e no desempenho muscular, leva à redução da autonomia evidenciada na realização das atividades de vida diária (AVDs) ${ }^{3}$. Além de apresentar correlação com índices de limitação funcional, a FM pode ser relacionada também a índices de morbidade e mortalidade ${ }^{5,6}$, independentemente da faixa etária. Portanto, a mensuração dos níveis de força é algo fundamental ${ }^{2,10}$, o que pode auxiliar no entendimento de inúmeros fatores relacionados diretamente com a diminuição da massa muscular em idosos9.

Atualmente, a avaliação da força por meio de dinamômetros é bastante utilizada por ser um procedimento de fácil aplicação $0^{2,3,12}$, que estabelece parâmetros confiáveis na mensuração da força muscular ${ }^{14}$.

Alguns autores ${ }^{17,18}$ afirmam que a utilização da dinamometria lombar (DL) na avaliação da força muscular do tronco é importante para estimar a capacidade física global, sobretudo em sujeitos que realizam grande esforço físico, como atletas ou trabalhadores em atividades ocupacionais que exijam maiores níveis de FM.

Em seu trabalho sobre a dinamometria isocinética de tronco em nadadores ${ }^{24}$, Secchi et al. afirmam que o desequilíbrio muscular que pode estar presente na região do tronco é considerado o principal fator causal de lesões. O tronco é importante para manter a estabilidade e o equilíbrio, principalmente quando os membros superiores desenvolvem movimentos de grande amplitude e alta velocidade. Assim, a DL possibilita a obtenção de dados sobre os níveis de força do tronco, constatando alterações ou desequilíbrios musculares, e podendo ser considerada um método utilizado para prevenção de lesões.

Estudando 50 mulheres com faixa etária entre $30 \mathrm{e}$ 50 anos, Guedes et al. ${ }^{15}$ buscaram comparar o efeito de diferentes frequências de treinamento de força sobre a força muscular isométrica da coluna lombar. Foi constatada uma força média de $62,40 \mathrm{kgf}( \pm 17,11)$ no grupo que realizava o treinamento de 2 a 3 vezes por semana, e 71,44 kgf $( \pm 15,21)$ no grupo que realizava treinamento 4 a 5 vezes por semana. Analisando esses valores, verificamos que, no presente estudo, as mulheres apresentaram níveis menores de força muscular lombar, com média de 48,50 $( \pm 18,80)$ kgf. Porém, esse valor pode ser relacionado à distinção no 
perfil amostral, sendo que abordamos indivíduos praticantes e não praticantes de atividades físicas, enquanto Guedes et al. avaliaram apenas mulheres que realizavam treinamento de força. Embora tenha sido utilizado um dinamômetro diferente do que utilizamos, o teste ocorreu de acordo com um procedimento de aplicação semelhante ao adotado em nossa pesquisa. Assim, podemos confrontar os resultados obtidos.

Ao realizar uma pesquisa sobre o nível de aptidão física em coletores de lixo do município de Vitória (ES), Araújo ${ }^{25}$ avaliou a força dorsal e de membros inferiores em 17 indivíduos. Foi constatada uma média de $143 \mathrm{kgf}( \pm 21,85)$, valores diferentes dos encontrados em nosso estudo média $114 \mathrm{kgf}( \pm 24,50)$. Essa discrepância de valores pode estar relacionada ao fato de que o nosso estudo teve como base um grupo amostral significativamente maior do que o estudado por Araújo, embora tenha sido utilizada metodologia semelhante na realização do teste de DL.

Reis-Filho et al. ${ }^{9}$ realizaram um estudo buscando correlacionar o estado nutricional com a força de preensão palmar, lombar e escapular em mulheres com idade entre $38 \mathrm{e}$ 71 anos, classificadas como fisicamente ativas. A amostra foi dividida em dois grupos, sendo um caracterizado como meia-idade e o outro como idosas. Foi obtida uma força lombar média de 44,60 kgf $( \pm 15,00)$ e 33,10 kgf $( \pm 11,30)$, respectivamente. Analisando apenas o grupo de meia-idade, que possui faixa etária mais semelhante com o grupo por nós estudado, percebemos valores semelhantes nos níveis de força muscular das mulheres, apenas com uma média de 3,90 kgf a mais de força na amostra do nosso estudo. No entanto, tais correlações podem ser questionadas pelo fato de terem sido empregados procedimentos metodológicos distintos na aplicação do teste de DL.

$\mathrm{Ni}$ et al. ${ }^{26}$ buscaram estabelecer dados normativos de força muscular da coluna vertebral lombar para a população chinesa sem dor nessa região. O estudo envolveu 120 pessoas, sem história de dor lombar durante os últimos 6 meses ou qualquer cirurgia prévia. Esses estudiosos concluíram que os homens são capazes de gerar um valor médio de força de extensão de cerca de $114 \%$ do seu peso corporal, enquanto as mulheres apresentaram um valor médio de $88 \%$. Foram encontrados como valores médios de contração isométrica voluntária máxima para extensão de tronco 23,65 kgf ( $\pm 4,91)$, em homens, e $14,29 \mathrm{kgf}( \pm 3,34)$, nas mulheres.

$O$ fato de ter sido utilizada metodologia distinta para coleta de dados da força lombar dificulta qualquer comparação com os dados obtidos em nossa pesquisa. Contudo, se mesmo assim confrontarmos com outros trabalhos, verificamos que os níveis de força mensurados em nosso estudo são os mais elevados.

Em outro estudo ${ }^{27}$, conduzido por Gonçalves e Barbosa, a força isolada dos eretores da coluna (região lombar) também foi avaliada por dinamometria numa instrumentação que permitia o posicionamento em decúbito ventral, isolando, assim, o movimento de extensão do tronco. Já Udermann, Mayer e Murray ${ }^{17}$ realizaram um estudo buscando quantificar a força isométrica de extensão lombar utilizando um dinamômetro Backup ${ }^{\mathrm{Tx}}$. Foram avaliados 60 indivíduos saudáveis de ambos os sexos, com idade média de 22,15 $( \pm 2,45)$ anos de idade. Constataram que a força média isométrica de extensão lombar variou de 39,39 ( $\pm 9,03)$ a 26,84 kgf $( \pm 7,30)$ nos homens, e de $25,37( \pm 8,46)$ a $16,06 \mathrm{kgf}( \pm 5,51)$ nas mulheres. A discussão dos resultados com ambos os trabalhos novamente é dificultada pela distinção nos procedimentos metodológicos empregados, sendo que Gonçalves e Barbosa avaliaram os pacientes em decúbito ventral e Udermann, Mayer e Murray utilizaram um dinamômetro com vários mecanismos de estabilização pélvica.

Um único estudo anterior que utilizou instrumentação e método semelhante ao nosso mostrou resultados parecidos em homens, mas divergiu dos valores encontrados em relação às mulheres ${ }^{2}$. Desta forma, nosso trabalho apresenta uma contribuição inicial na determinação dos valores normativos do teste de DL.

Esse teste dinamométrico pode ser útil no processo avaliativo ocupacional, bem como em atletas e pacientes com distúrbios do aparelho locomotor, em especial aqueles envolvendo o tronco.

Percebemos que qualquer tentativa de discutir os resultados deste estudo ficou limitada pela escassez de outros trabalhos utilizando metodologia semelhante. Assim, também não foram encontrados valores normativos para o teste de DL.

Parece fundamental esclarecer que os valores obtidos em testes dinamométricos devem considerar fatores inerentes às populações estudadas ${ }^{2}$. O Brasil, um país continental, apresenta grande diversidade étnica, regiões com características bastante distintas e, portanto, os resultados desse estudo devem ser considerados no âmbito da Região Sul, em especial do município de Joinville e microrregião, Estado de Santa Catarina. 
Cabe lembrar que os valores aqui sugeridos devem ser vistos com cautela quando da sua generalização para outras partes do país. As várias regiões em todo o território nacional apresentam população com influências étnicas bastante distintas, sendo que vários fatores culturais podem exercer influências diretas nos níveis de FM.

\section{CONCLUSÕES}

Como ainda não existem valores normativos bem estabelecidos para o teste de dinamometria lombar, este trabalho representa uma contribuição inicial. Os valores mensurados são preliminares e específicos para essa região do estado de Santa Catarina.

Vários fatores dificultam o estabelecimento de valores normativos para o teste de DL, sendo que o principal deles é a diversidade de procedimentos metodológicos empregados para a avaliação da força muscular lombar. Algumas características do grupo amostral - como faixa etária e perfil sociodemográfico e cultural — também devem ser levadas em consideração, pois podem influenciar nos resultados para a dinamometria lombar.

As informações deste estudo podem contribuir para a área da reabilitação física, esportiva e da ergonomia, pois trata-se de uma medida rápida, de fácil aplicação e de baixo custo.

$\mathrm{Na}$ área da reabilitação, a avaliação da força muscular por meio da dinamometria pode ser útil no estabelecimento de objetivos mais claros no processo de reabilitação de pacientes, facilitando o estabelecimento de critérios de alta. Já na área esportiva, esse procedimento pode ser utilizado como método de prevenção de lesões, principalmente nos atletas praticantes de atividades que exigem altos níveis de força muscular de tronco. No campo da ergonomia e da saúde ocupacional, essas informações podem contribuir no desenvolvimento de produtos e de equipamentos, além da possível inserção desse teste no processo admissional, bem como nos exames periódicos de trabalhadores em ocupações que exigem maior utilização do tronco.

\section{REFERÊNCIAS}

1. Hall S. Biomecânica básica. 6. ed. Rio de Janeiro: Guanabara Koogan, 2013

2. Soares AV, Carvalho-Júnior JM, Fachini J, Domenech SC, BorgesJúnior NG. Correlação entre os testes de dinamometria de preensão manual, escapular e lombar. Rev Acta Bras Movimento Humano. 2012;2(1):65-72.

3. Oliveira FB, Moreira D. Força de preensão palmar e diabetes mellitus. Rev Bras Clin Med. 2009;7:251-5.

4. Barbosa AR, Souza JMP, Lebrão ML, Marucci MFN. Relação entre estado nutricional e força de preensão manual em idosos do município de São Paulo, Brasil: dados da pesquisa SABE. Rev Bras Cineantropom Desempenho Hum. 2006;8(1):37-44.

5. Brill PA, Macera CA, Davis DR, Blair SN, Gordon N. Muscular strength and physical function. Med Sci Sports Exerc. 2000;32(2):412-6

6. Ruiz JR, Sui X, Lobelo F, Morrow-Júnior JR, Jackson AW, Sjöström $\mathrm{M}$, et al. Association between muscular strength and mortality in men: prospective cohort study. BMJ. 2008;337:a439.

7. Sobral MLP, Badessa MPSG, Sobral MLP, Oliveira-Júnior JB. Estudo da prevalência de algias na coluna vertebral em residentes de cirurgia cardiovascular: estudo inicial. Rev Bras Med Trab. 2013;11(2):82-9.

8. Haeffner R, Sarquis LMM, Haas GFS, Heck RM, Jardim VMR. Prevalência de lombalgia e fatores associados em trabalhadores de uma empresa agropecuária do sul do Brasil. Rev Bras Med Trab. 2015;13(1):35-42.
9. Reis-Filho AD, Santini E, Neves T, Fett WCR, Fett CA. Análise do estado nutricional e da força de preensão palmar, lombar e escapular em mulheres de meia idade e idosas. Brazilian Journal of Biomotricity. 2012;6(4):245-53.

10. Souza LAC, Martins JC, Teixeira-Salmela LF, Godoy MR, Aguiar LT, Faria CDCM. Avaliação da força muscular pelo teste do esfigmomanômetro modificado: uma revisão da literatura. Fisioter Mov. 2013;26(2):437-52.

11. Amaral JF. Medição da força muscular máxima de preensão da mão com três diferentes dinamômetros. Juiz de Fora. Monografia [Graduação em Bacharel em Educação Física] - Universidade Federal de Juiz de Fora; 2010.

12. Reis MM, Arantes PMM. Medida da força de preensão manual validade e confiabilidade do dinamômetro Saehan. Fisioter Pesq 2011:18(2):176-81.

13. Bohannon RW. Reference values for extremity muscle strength obtained by hand-held dynamometry from adults aged 20 to 79 years. Arch Phys Med Rehabil. 1997;78(1):26-32.

14. Moura PMLS. Estudo da força de preensão palmar em diferentes faixas etárias do desenvolvimento humano. Brasília. Dissertação [Mestrado em Ciências da Saúde] -Universidade de Brasília; 2008

15. Guedes WA, Farias DL, Tibana RA, Nascimento DC, Vieira, DCL, Tajra V, et al. Efeito de diferentes frequências de treinamento sobre a força muscular isométrica da coluna lombar em mulheres praticantes de treinamento de força. Educação Física em Revista. 2013;7(1):1-10. 
16. Heyward VH. Avaliação e prescrição de exercício: técnicas avançadas. 4. ed. Porto Alegre: ArtMed, 2004.

17. Udermann BE, Mayer JM, Murray SR. Quantification of isometric lumbar extension strength using a BackUP lumbar extension dynamometer. Res Q Exerc Sport. 2004;75(4):434-9.

18. Santos LJM. Dinamometria isocinética lombar. Revista Digital Efdeportes, Buenos Aires. 2002; ano 8(49). Disponível em: <http:// www.efdeportes.com/efd49/dinam.htm>. Acesso em: 26 mar. 2015.

19. Barbosa L, Gomes EB, Carvalho GA, Pinheiro HA. Efeitos da imersão em gelo na força de preensão palmar em adultos jovens. Acta Fisiatr. 2013;20(3):138-41.

20. Queiroz JCF. Correlação entre a força de preensão palmar e a força da musculatura respiratória em indivíduos obesos e não obesos. Brasília. Dissertação [Mestrado em Saúde e Atividade Física] Universidade Católica de Brasília; 2006.

21. Carreira H, Amaral TF, Brás-Silva C, Oliveira BMPM, Borges N. Força da preensão da mão numa amostra de crianças dos 11 aos 14 anos. Acta Med Port. 2010;23(5):811-8.

22. Fernandes AA, Marins JCB. Teste de força de preensão manual: análise metodológica e dados normativos em atletas. Fisioter Mov. 2011;24(3):567-78.
23. Santos MM, Ferreira AHS, Costa MC, Guimarães FJS, Ritti-Dias RM. Contribuição da massa muscular na força de preensão manual em diferentes estágios maturacionais. ConscientiaeSaúde. 2011;10(3):487-93.

24. Secchi LLB, Muratt MD, Andrade NVS, Greve JMD. Dinamometria isocinética de tronco em nadadores de diferentes estilos. Acta Ortop Bras. 2010;18(5):295-7.

25. Araújo DZM. Avaliação do nível de aptidão física em coletores de lixo domiciliar do município de Vitória/ES. Revista Brasileira de Prescrição e Fisiologia do Exercício. 2011;5(28):347-54.

26. Ni GX, Luk KDK, Lu WW, Cheng ACS, Cheung KMC, Tong FCW, et al. Normative data of lumbar spinal dynamic function for Chinese people without low back pain. Hong Kong Journal of Orthopaedic Surgery. 2003;7(1):25-32.

27. Gonçalves M, Barbosa FSS. Análise de parâmetros de força e resistência dos músculos eretores da espinha lombar durante a realização de exercício isométrico em diferentes níveis de esforço. Rev Bras Med Esporte. 2005;11(2):109-14.

Endereço para correspondência: Fernando Luís Fischer Eichinger Rua Madeira, 258 - Guanabara - CEP: 89207-790 - Joinville (SC), Brasil E-mail: fernando_Ife@hotmail.com 